\title{
A Nursing Perspective on Seclusion in Australia's Mental Health System
}

\author{
Jieli LI* \\ Bachelor Degree of Medicine, China \\ *Corresponding author: Jieli LI, bachelor's degree of Medicine, China
}

\begin{tabular}{|c|c|}
\hline ARTICLE INFO & ABSTRACT \\
\hline Received: 蔧 May 06, 2019 & Abbreviations: DHHS: Department of Health and Human Services; NDIS: Nati \\
\hline Published: 幽 May 14, 2019 & $\begin{array}{l}\text { Disability Insurance Scheme; NMHCCF: National Mental Health Consumer \& Carer Forum; } \\
\text { IM: Intramuscularwhen; IV: Intravenous }\end{array}$ \\
\hline
\end{tabular}

Citation: Jieli LI. A Nursing Perspective on Seclusion in Australia's Mental Health System. Biomed J Sci \& Tech Res 18(1)-2019. BJSTR. MS.ID.003089.

\section{Introduction}

It is now widely agreed that the use of restrictive intervention such as seclusion in mental health service can impair therapeutic trust, infringe human rights and traumatise or re-traumatise consumers [1]. Seclusion was defined as the solitary confinement of a person to an enclosed space from which it is not within the ability of the person confined to exit [2]. In Victoria, the application of seclusion is strictly regulated by the Mental Health Act 2014. Seclusion can only be taken as "the last resort" when other less restrictive choices have been attempted and proven to be unhelpful in a situation (Mental Health Act 2014 (Vic), s. 102 [3]. Healthcare professionals are encouraged to take prevention and early interventions to diminish and wherever possible eradicate the practice of seclusion [2]. The aim of this paper is to discuss the relevant mental health nursing practices regarding the procedure of seclusion which includes legal documentation, environmental preparation, clinical monitoring and review, meeting the person's needs, and timely notification. It will also explore different views about the use of seclusion from recent literature. Lastly, an alternative approach to seclusion will be proposed with supporting evidence.

According to the Mental Health Act 2014, prior to any type of restrictive intervention, the authority for use should be obtained from an authorised psychiatrist or delegate. A registered medical practitioner or a senior registered nurse on duty can initiate seclusion but the authorised psychiatrist or delegate must be notified as soon as practicable (Mental Health Act 2014 (Vic)) [3]. It is also required that the authorised psychiatrist or delegate perform soonest examination on the secluded person to decide whether continued use of seclusion is necessary unless the person has been released in the meantime (Mental Health Act 2014 (Vic)) [3]. In terms of documentation, the Restrictive interventions - authority for use MHA 140 form needs to be completed at the commencement of seclusion (Mental Health Act 2014 (Vic)) [3]. In addition, a written report must be made to the chief psychiatrist regarding their use of seclusion on a patient (Mental Health Act 2014 (Vic)) [3]. If a restrictive intervention is utilized in a disability service, a report must be made to the Senior Practitioner Team under Department of Health and Human Services (DHHS) in the support of the National Disability Insurance Scheme (NDIS) (DHHS, 2016). For environmental preparation, evidence suggests that seclusion ought to only take place in an environment designed expressly for that purpose [4]. A room supports prevention or minimization is essential for the safe delivery of seclusion when it is necessary or inevitable [5]. For example, protective walls, an equipped door, and high-performance sound-absorbing ceiling tiles and flooring are recommended for the stimuli reduction and patient protection [4]. Before proceeding to seclusion, assessment should be conducted including physical check-ups, assessment of pre-existing diseases, the urgency of immediate medical attention, and the risk of selfharming in seclusion room [4]. During seclusion, clinical reviews 
on the secluded person must be conducted by a registered nurse or registered medical practitioner as often as is appropriate but not less frequently than every 15 minutes (Mental Health Act 2014 (Vic)) [6].

The authorised psychiatrist or delegate must medically examine the person at least every 4 hours (Mental Health Act 2014 (Vic)) [3]. The reviewing clinician needs to evaluate the vital signs, food and fluid intake and excretions, mental state and behaviour of the secluded person and complete the Restrictive interventions observations MHA 142 form (Mental Health Act 2014 (Vic)) [3]. It is important to ensure the provision of necessities such as food and drinks; appropriate supplies such as bedding, clothing, and toiletries to the patient (Mental Health Act 2014 (Vic)) [3]. The person's dignity and privacy needs to be protected (Mental Health Act 2014 (Vic)) [3]. If the use of seclusion is no longer necessary, the person must be immediately released (Mental Health Act 2014 (Vic)) [3]. A parent, a guardian, or a nominated person has legal right to be notified of the use of seclusion as soon as practicable (Mental Health Act 2014 (Vic)) [3].

If they are not satisfied with the mental health services, a complaint against their healthcare providers can be made to the Mental Health Complaints Commissioner (Mental Health Act 2014 (Vic)) [3]. Having discussed the process of seclusion and the relevant nursing practices, the following section will explore different opinions supporting or opposing the practice of seclusion from recent literature. In Australia, there is a trend of increasing awareness that restrictive intervention causes short- and longterm emotional destruction which highlights a failure in care and treatment [7]. Seclusion is frequently linked with human rights abuse and is not "evidence-based therapeutic interventions" (National Mental Health Consumer \& Carer Forum (NMHCCF), 2009) [8]. The NMHCCF in Australia believes that involuntary seclusion and restraint are currently operated at "unacceptably high levels" and therefore must be removed from practice in Australia's mental health services (NMHCCF, 2009) [8]. The Australian College of Mental Health Nurses (ACMHN) [1] further supported with a Seclusion and Restraint Position Statement in 2016 that "seclusion and restraint use should be reduced and ultimately ended" (ACMHN, 2016). Scholars from Australia and international wide question the use of restraint and seclusion in patient behaviour control seems to be unethical $[9,10]$. An Australian survey conducted in the psychiatric inpatient setting and emergency departments reported that $37.7 \%$ of nurses agree and $23.44 \%$ of nurses strongly agree that seclusion violates the autonomy of the consumer [8]. There has been continued cries for the reduction and elimination of seclusion which is reflected in Australian government policy directives and international clinical initiatives [11-14].

Despite the phenomenal growth of evidence and government policies against seclusion, studies about the advantages of seclusion can also be found, though very little. One cohort study reported that some participants considered the use of seclusion was helpful in terms of "answering the need for calm, sleep, and safety" [14]. Healthcare professionals have achieved successfully limit setting effect and prevented episodes of violence to self and others [12,13]. Similarly, in the study of Haw, Stubbs, Bickle and Stewart [15], 16\% of participants reported their last episode of seclusion was positive and helpful. Their reply was "you can get away from everything for a while" [9]. They responded that "sometimes it's nice to be on your own and not to have people around when you are feeling upset" [9]. They also spoke of seclusion having a positive effect on thoughts and emotions "you can chill out, like get peace of mind" [9]. It provides a time for quiet reflection that "you reflect back on what happened and apologise" [9].

Although some claimed their experience of seclusion evoked many unpleasant thoughts and emotions which re-traumatized them, they commented more on the "practice of seclusion" rather than "seclusion itself". For example, they said "the staff treat me like an animal"; "they deliberately punishing me"; "they acted unprofessionally by using of undue force"; "you are stuck in a room like a cell with no ventilation, no window, no fan, no bed often, nothing and being held down on the flood, then dragged away" [9]. They complained about the cold, claustrophobic and uncomfortable seclusion room and the fact that they have not been debriefed about what's going on for them [9]. That is to say, the poor practice of seclusion contributes to consumer's negative experience and opposing attitude towards seclusion. It cannot change the fact in a study that $38.87 \%$ of nurses agree and $40.63 \%$ of nurses strongly agree that the use of seclusion is necessary as protection in dangerous situations [8]. In summary, although the disadvantages of seclusion have been widely recognised far more outweigh its advantages which has been validated by government policies, there are still clinicians and patients who favour seclusion [12]. If seclusion is unavoidable, the standardised procedure explained in the previous section should be followed to minimise the negative effects on a secluded person.

According to Mental Health Act 2014 (Vic) [16], seclusion can only be an option for a person after all non-traumatic and less restrictive alternatives have been attempted or considered but proven to be unhelpful. Vieta et al. [16] recommended an effective intervention which leads to eliminating coercive measures: verbal de-escalation. Rishmond et al. [17] further corroborate the idea of attempting verbal de-escalation in a greater part of agitated patients in acute emergency settings. Participants in a cohort study strongly suggest that de-escalation techniques should be used more often before secluding them: "try to talk to me first before going into seclusion" [9]. The intentions of verbal de-escalation are: establishing a therapeutic relationship with patients; enhancing the patients' capability of self-control; introducing clear limit setting; reducing the aggressiveness and hostility; and preventing a probable episode of violence [16] 
Major domains of de-escalation are: respecting patient's personal space; not being provocative; coaching the patient selfcontrol skills; setting reasonable limit in a respectful manner; and be optimistic and provide hope Rishmond et al. [17] There are a variety of verbal de-escalation techniques which have been testified successfully to reduce agitation and lessen potential violence [16]. For instance: talking to the patient in a relaxed, gentle, assured tone; answering calmly with a firm attitude; being flexible in the dialogue; using open-ended questions; and reserving your own judgement [16]. Accumulative studies have promoted verbal de-escalation as the way to enhance patients' self-control while building trust with healthcare providers which in return can lead to patient's confidence of seeking help earlier to avoid restrictive interventions Richmond et al. (2012). In summary, before reaching the episode of violent behaviour, early intervention and prevention techniques such as verbal de-escalation can be valuable, therapeutic and effective in eliminating seclusion.

However, if an agitated person has already passed the stage of being intervened by preventive techniques and sits at the peak of a crisis, restrictive interventions might be unavoidable [12] An alternative approach to seclusion proposed in this paper is pharmacologic intervention. Some practitioners refer it to chemical restraint (DHHS, 2016). It is not a form of treatment. Rather, the primary purpose of using the medication is to control a person's behaviour (DHHS, 2013). Given during a violent crisis, a specific scenario would be a " 250 -pound male psychotic patient is throwing furniture towards co-patients and nursing staff members", chemical restraint might be recommended at this point to control the situation [16]. The appropriate pharmacologic agent can calm agitation, assist patient to concentrate, enable easier access to interpersonal interventions [16]. There are three types of chemical restraint administration: routine medication; PRN medication; and in an emergency (DHHS, 2016). Pharmacologic agents that are typically used for chemical restraint are ketamine, typical and atypical antipsychotics, and benzodiazepines [18]. Medication doses and the dosing interval can be affected by the patient's body size, age, level of agitation, previous response to sedative drugs and his or her medical history [18]. Most frequent administration route is Intramuscular (IM) when intravenous (IV) access is not clinically safe to perform [18]. Interestingly, a positive response was found among healthcare professionals towards chemical restraint compared to seclusion [12]. In the study of Haw et al [9], the survey result also shows that most participants (patients) preferred intra-muscular medication which is a way of chemical restraint to seclusion. These results are in line with Dr. Jeffrey Keller' opinion, who is a Board Certified Emergency Physician with 25-year emergency medicine practice experience. He believes that chemical sedation is a community standard of care, which is safer than prolonged physical restraint and carries less legal risk than does prolonged physical restraint [19]. Nevertheless, cautions should be exercised when initiating pharmacological intervention
[18]. The primary goal of pharmacologic agent administration is to rapidly and safely manage patient's agitated behaviour without overly sedating them [16]. If large doses of benzodiazepines or tranquilizers are regularly given to sedate consumers against their will, restrict their freedom, actively placing them in chemical restraint, the end result can be equalled to or even worse than seclusion in that consumers are unable to function and they are not in control of the situation [18].

What is more, added medication side-effects can post a potential danger to patients especially those who have pre-existing medical conditions [18]. To conclude, growing evidence has shown the negative consequences of practicing seclusion. Seclusion should only be utilised as the last resort in mental health services. Where possible, early intervention and prevention techniques should be implemented to minimise and eliminate the use of seclusion [20]. Verbal de-escalation is one of the techniques that has been testified to be valuable, therapeutic and effective in seclusion reduction. In the situation where seclusion is clinically necessary to prevent harm and is permitted under legislation, healthcare providers need to follow the procedure of safe application of seclusion which is regulated by state and territory legislation and mandatory policy [21-23]. Additionally, this essay has discussed the relevant practice of chemical restraint which can be an alternative approach to seclusion in some cases and might have a better outcome when used with cautions.

\section{References}

1. Kinner S, Harvey C, Hamilton B, Brophy L, Roper C, et al. (2017) Attitudes towards seclusion and restraint in mental health settings: Findings from a large, community-based survey of consumers, carers and mental health professionals. Epidemiology and Psychiatric Sciences 26(5): 535544.

2. Mckenna B, Mcevedy S, Maguire T, Ryan J, Furness T (2017) Prolonged use of seclusion and mechanical restraint in mental health services: A state-wide retrospective cohort study. International Journal of Mental Health Nursing, 26(5): 491-499.

3. Mental Health Act 2014 (Vic).

4. Kaar S, Walker H, Sethi F, McIvor R (2017) The function and design of seclusion rooms in clinical settings. Journal of Psychiatric Intensive Care 13(2): 83-91.

5. (2012) Office of Disease Prevention and Health Promotion. Secure rooms and seclusion standards and guidelines: A literature and evidence review.

6. Bhavsar V, Sethi F, Hillier B (2014) Medical guidelines for PICU seclusion reviews. Journal of Psychiatric Intensive Care 10(1): 40-50.

7. Bellenger E, Ibrahim J, Kennedy B, Bugeja L (2019) Prevention of physical restraint use among nursing home residents in Australia: The top three recommendations from experts and stakeholders. International Journal of Older People Nursing 14(1): 1-9.

8. (2009) Ending seclusion and restraint in Australian mental health services. National Mental Health Consumer \& Carer Forum (NMHCCF).

9. Bowers L, James K, Quirk A, Simpson A, Sugar Stewart D (2015) Reducing conflict and containment rates on acute psychiatric wards: The safewards cluster randomised controlled trial. International Journal of Nursing Studies 52(2015): 1412-1422.

10. Gerace A, Muir Cochrane E (2019) Perceptions of nurses working with psychiatric consumers regarding the elimination of seclusion and 
restraint in psychiatric inpatient settings and emergency departments: An Australian survey. International Journal of Mental Health Nursing 28(1): 209-225.

11. (2018) Australian Institute of Health and Welfare. Mental health services in Australia-Restrictive practices.

12. (2016) The Royal Australian \& New Zealand College of Psychiatrists. Minimising the use of seclusion and restraint in people with mental illness.

13. (2017) Strategies to end the use of seclusion, restraint and other coercive practices: WHO quality rights training to act, unite and empower for mental health (pilot version). World Health Organization.

14. Larue C, Dumais A, Boyer R, Goulet MH, Bonin JP, et al. (2013) The experience of seclusion and restraint in psychiatric care settings: Perspectives of the patients. Issues in Mental Health Nursing, 34(2): 317-324.

15. Haw C, Stubbs J, Bickle A, Stewart I (2011) Coercive treatments in forensic psychiatry: A study of patients' experiences and preferences. Journal of Forensic Psychiatry \& Psychology 22(4): 564-585.

16. Vieta E, Garriga M, Cardete L, Bernardo M, Lombrana M, (2017) Protocol for the management of psychiatric patients with psychomotor agitation. BioMedCentral Psychiatry 17(328): 1-11.

ISSN: 2574-1241

DOI: 10.26717/BJSTR.2019.18.003089

Jieli LI. Biomed J Sci \& Tech Res

(C) This work is licensed under Creative

Submission Link: https://biomedres.us/submit-manuscript.php
17. Richmond JS, Berlin JS, Fishkind AB, Holloman GH, Zeller SL, et al. (2012) Verbal de-escalation of the agitated patient: consensus statement of the America association for emergency psychiatry project BETA deescalation workgroup. The West Journal of Emergency Medicine 13(1): 17-25.

18. Macdonald RD, Albulushi S (2017) Articles that may change your practice: Chemical restraint of agitated patients. Air Medical Journal 36(3): 101-104

19. Keller J (2013) Chemical sedation is safer than prolonged physical restraint. Jail Medicine.

20. (2016) Australian College of Mental Health Nurses (ACMHN), Seclusion and restraint position statement.

21. (2013) Chemical restraint chief forensic psychiatrist clinical guideline.

22. (2016) Senior practitioner report. Health and Human Services 20152016.

23. Valimaki M, Lorig KR, Anttila M, Lantta T, Adams CE (2017) Study protocol for a cluster randomised controlled trial to assess the effectiveness of user-driven intervention to prevent aggressive events in psychiatric services. BioMedCentral Psychiatry 17(132): 1-13.

$\begin{array}{ll}\text { BIOMEDICAL } & \text { Assets of Publishing with us } \\ \text { RESEARCHES } & \text { - Global archiving of articles } \\ & \text { - Immediate, unrestricted online access } \\ & \text { - Rigorous Peer Review Process } \\ \end{array}$

